\title{
Treatment of experimental non-alcoholic steatohepatitis by targeting $\alpha 7$ nicotinic acetylcholine receptor-mediated inflammatory responses in mice
}

\author{
ZHOU ZHOU ${ }^{1 *}$, YING-CHAO LIU ${ }^{2 *}$, XIAO-MEI CHEN ${ }^{3}$, FU-QIANG LI $^{3}$, \\ XIAO-JUAN TONG ${ }^{3}$, YUE-PING DING ${ }^{4}$ and CUI-LAN TANG ${ }^{3}$
}

\begin{abstract}
${ }^{1}$ Department of Anesthesiology, The Second Clinical Medical College, Zhejiang Chinese Medical University, Hangzhou, Zhejiang 310053; Departments of ${ }^{2}$ Digestive Diseases, ${ }^{3}$ Infectious Disease and ${ }^{4}$ Intensive Care Unit, The Second Affiliated Hospital, Zhejiang Chinese Medical University, Hangzhou, Zhejiang 310005, P.R. China
\end{abstract}

Received October 20, 2014; Accepted June 26, 2015

DOI: $10.3892 / \mathrm{mmr} .2015 .4318$

\begin{abstract}
Non-alcoholic fatty liver disease (NAFLD) is one of the most common types of liver disease, affecting up to $30 \%$ of the general population worldwide. Non-alcoholic steatohepatitis (NASH) is a severe form of NAFLD without any effective therapies available. The present study showed that activation of $\alpha 7$-nicotinic acetylcholine receptor ( $\alpha 7 \mathrm{nAChR})$ may be a novel potential strategy for NASH therapy. Treatment with the $\alpha 7 \mathrm{nAChR}$ agonist nicotine for three weeks obviously attenuated hepatic steatosis in a high-fat diet-induced mouse model of NASH. Investigation of the underlying mechanism showed that nicotine reduced the secretion of the pro-inflammatory cytokines tumor necrosis factor $\alpha$ and interleukin 6 in vitro and in vivo. Inflammation is an integral part of NASH and is the most prevalent form of hepatic pathology found in the general population; therefore, the effect of $\alpha 7 \mathrm{nAChR}$ activation against NASH may be ascribed to its anti-inflammatory effects. In addition, the present study showed that nicotine-stimulated $\alpha 7 \mathrm{nAChR}$ activation led to a significant downregulation of nuclear factor kappa B (NK-кB) and extracellular signal-regulated kinase (ERK). It therefore appeared that activation of $\alpha 7 \mathrm{nAChR}$ suppressed the production of pro-inflammatory

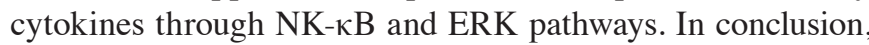
the present study indicated that targeting $\alpha 7 \mathrm{nAChR}$ may represent a novel treatment strategy for NASH.
\end{abstract}

Correspondence to: Dr Cui-Lan Tang, Department of Infectious Disease, The Second Affiliated Hospital, Zhejiang Chinese Medical University, 318 Chao-Wang Road, Hangzhou, Zhejiang 310005, P.R. China

E-mail: 1747603542@qq.com

${ }^{*}$ Contributed equally

Key words: non-alcoholic fatty liver disease, non-alcoholic steatohepatitis, $\alpha 7$-nicotinic acetylcholine receptor, inflammation, tumor necrosis factor $\alpha$, interleukin 6, nuclear factor kappa B

\section{Introduction}

Non-alcoholic fatty liver disease (NAFLD) is a cause of fatty liver, occurring when fat is deposited in the liver (steatosis) not due to excessive alcohol use. It is associated with insulin resistance and metabolic syndrome (1). NAFLD is currently considered to be the most common cause of chronic liver disease worldwide (2) and associated with other potentially life-threatening diseases and increased mortality from cardiovascular diseases, malignancy and hepatic complications. NAFLD has also been found to be associated with several extra-hepatic disorders, including breast cancer, polycystic ovary syndrome and renal dysfunction (3-15). NAFLD encompasses a wide spectrum of liver diseases ranging from simple steatosis to non-alcoholic steatohepatitis (NASH) (1), which is the most extreme form of NAFLD and is regarded as a major cause of cirrhosis of the liver of unknown cause (16). NASH is a major health problem and complicated by portal hypertension and hepatic decompensation, and is occasionally accompanied with hepatocellular carcinoma (HCC) (17).

Recently, various treatment modalities have been applied in NASH, including lifestyle modification, surgical intervention and pharmacological agents (including insulin sensitizers, anti-oxidant agents, lipid-lowering agents and tumor necrosis factor-alpha (TNF- $\alpha$ ) antagonists) (18-20). However, to date, there are no US Food and Drug Administration-approved medical therapies for NASH or liver fibrosis. There is an urgent requirement for novel therapeutic approaches $(17,21)$. As inflammatory activation has a significant role in NASH progression, anti-inflammatory therapy for NASH is of increasing interest (22). For example, TNF- $\alpha$ antagonist pentoxifylline, interleukin (IL)-6 antagonist Sant7 and the TNF- $\alpha$-specific monoclonal antibodies infliximab, adalimumab and certolizumab have been studied in a number of clinical NAFH trials (23). At present, anti-inflammatory strategies for NASH are restricted to targeting one single cytokine, e.g., IL-1 receptor, IL- 6 or TNF- $\alpha$. However, multiple cytokines are involved in the inflammatory response of NASH. Therefore, targeting an upstream signaling molecule that regulates multiple cytokine production may improve the objective 
response rates (24). Recently, a novel neural pathway termed as cholinergic anti-inflammatory reflex, has been discovered, which inhibits the production of inflammatory cytokines and may be a novel anti-inflammatory strategy for NASH.

The $\alpha 7$-nicotinic acetylcholine receptor $(\alpha 7 \mathrm{nAChR})$ is a sub-type of nicotinic acetylcholine receptor and has a crucial role in mediating the cholinergic anti-inflammatory signaling pathway (25). It is expressed on different types of cells, including neurons, macrophages, lymphocytes, monocytes and dendritic cells. Activation of the $\alpha 7 \mathrm{nAChR}$ expressed on resident macrophages may suppress the local inflammation by reducing the production of pro-inflammatory cytokines TNF- $\alpha$ and IL-6, which are closely associated with certain inflammatory diseases, including sepsis, rheumatoid arthritis, asthma and diabetes (26). It is therefore indicated that $\alpha 7 \mathrm{nAChR}$ is a promising target for developing novel anti-inflammatory drugs. However, to date, it has remained to be clarified whether $\alpha 7 \mathrm{nAChR}$ is associated with NASH.

The present study assessed whether activation of $\alpha 7 \mathrm{nAChR}$ was able to prevent the progression of NASH, and whether targeting of $\alpha 7 \mathrm{nAChR}$ may represent a novel strategy for NASH therapy.

\section{Materials and methods}

Cell culture and reagents. RAW 264.7 cells were obtained from the American Type Culture Collection (Manassas, VA, USA) and maintained in Dulbecco's modified Eagle's medium (Gibco-BRL, Invitrogen Life Technologies, Carlsbad, CA, USA) supplemented with $10 \%$ heat-inactivated fetal bovine serum (Invitrogen Life Technologies) in a humidified atmosphere of $95 \%$ air with $5 \% \mathrm{CO}_{2}$ at $37^{\circ} \mathrm{C}$. Nicotine was purchased from Sigma-Aldrich (St. Louis, MO, USA; n=80).

Experimental protocols and animals. C57 male mice at four weeks of age (weight, 17-23 g) were purchased from the Model Animal Research Center of Nanjing University (Nanjing, China) and housed in the laboratory animal center of Zhejiang Chinese Medical University (Hangzhou, China) at $22^{\circ} \mathrm{C}$ with a 12 -h light/dark cycle. Mice were randomly divided into four groups $(n=10)$ and fed either a control diet $(10 \% \mathrm{kcal}$ as fat; Mediscience Ltd., Yangzhou, China) or a high-fat diet (HFD; 60\% kcal as fat; Medicience Ltd) for 18 weeks with or without nicotine for three weeks: 1) Control group, mice were fed a control diet and supplemented with normal saline; (2) HFD group, mice were fed a HFD and supplemented with normal saline; (3) control + nicotine $5 \mathrm{mg} / \mathrm{kg}$ group, mice were fed a control diet and supplemented with nicotine at a dose of $5 \mathrm{mg} / \mathrm{kg}$; (4) HFD + nicotine $5 \mathrm{mg} / \mathrm{kg}$ group, mice were fed a HFD and supplemented with nicotine at the dose of $5 \mathrm{mg} / \mathrm{kg}$. During the 18 weeks of feeding, the body weight was measured every week. At the end of the experiment, mice were sacrificed by cardiac puncture under $\mathrm{CO}_{2}$ anesthesia, and livers were collected for further analysis.

All animals used in the present study were housed and cared for in accordance with the Chinese Pharmacological Society Guidelines for Animal Use. The protocols of the present study were approved by the Committee on the Ethics of Animal Experiments of the Zhejiang Chinese Medical University (Hangzhou, China; permit no. 2012-1849). All surgeries were performed under sodium pentobarbital anesthesia $(70 \mathrm{mg} / \mathrm{kg}$; Sigma-Aldrich) and all efforts were made to minimize suffering.

Biochemical serum analysis. The activity levels of aspartate aminotransferase (AST) and alanine aminotransferase (ALT) (10) were determined using an automatic blood chemical analyzer (Dry-Chem 4000i; Fujifilm, Tokyo, Japan). TNF- $\alpha$ and IL-6 levels were measured using ELISA kits (cat. nos. EK0527 and EK0441; Boster Biological Inc., Wuhan, China).

Histological examination and Oil Red $O$ staining. The fixed liver tissue was cut into 3-mm blocks, which were embedded in paraffin and cut into $4-\mu \mathrm{m}$ slices. After being de-paraffinized using xylene and ethanol dilutions and re-hydration, the sections were stained with hematoxylin and eosin (H\&E; Bogoo, Shanghai, China) to examine the tissue structure, inflammatory cell infiltration, necrosis and lipid accumulation.

For Oil Red O staining, cryosections of optimal cutting temperature compound-embedded liver tissues $(10 \mathrm{~mm})$ were fixed in $10 \%$ buffered formalin for $5 \mathrm{~min}$ at room temperature, stained with Oil Red O (Biohao Company, Wuhan, China) for $1 \mathrm{~h}$, washed with $10 \%$ isopropanol and then counterstained with hematoxylin for $30 \mathrm{sec}$. A Nikon E600 microscope (Nikon, Tokyo, Japan) and Leica Application Suite (Leica Microsystems, Inc., Buffalo Grove, IL, USA) were used to capture images of the Oil Red O-stained tissue sections at 40x magnification.

Isolation of macrophages from liver tissue. Forty normal, healthy mice were anesthetized and liver tissues were perfused in situ via the superior vena cava with a perfusion buffer (13 Hanks' balanced salt solution; Gino Biological Medical Technology, Co., Ltd., Hangzhou, China), followed by a digestion buffer [13 Hanks' balanced salt solution, supplemented with $0.05 \%$ collagenase (Type IV; Sigma-Aldrich), $1.25 \mathrm{mmol} / 1 \mathrm{CaCl}_{2}, 4 \mathrm{mmol} / \mathrm{I} \mathrm{MgSO}_{4}$ and $10 \mathrm{mmol} / 1$ 4-(2-hydroxyethyl)-1-piperazineethanesulfonic acid]. The resulting cell suspension was filtered through a sterile $100-\mathrm{mm}$ nylon mesh (Solarbio, Beijing, China) and centrifuged at $50 \mathrm{xg}$ to selectively sediment hepatocytes from non-parenchymal cells (NPCs). The pellet of hepatocytes was re-suspended and subsequently washed two more times with centrifugation at $50 \mathrm{xg}$. The NPCs in the first and second supernatants from the low-speed centrifugations were pelleted by high-speed centrifugation $(1,300 \mathrm{x}$ g), followed by re-suspension in a small volume prior to isopycnic sedimentation in Percoll as previously described (27). Cell viability (90\%) was determined by trypan blue exclusion (Sigma-Aldrich). The Kupffer cells were treated with lipopolysaccharide (LPS; Escherichia coli O111:B4; Sigma-Aldrich; $100 \mathrm{nM}$ ) for 16-18 h, following which the culture medium was replaced with medium without serum, and in the presence or absence of nicotine (concentrations between 0 and $10 \mu \mathrm{m}$ ) for $6 \mathrm{~h}$. Treatment with $\alpha$-bungarotoxin ( $\alpha$-BGT; Zhongxin Dongtai Company, Laiyang, China) was also performed.

Western blot analysis. Whole-cell lysates were prepared using radioimmunoprecipitation assay lysis buffer (Beyotime Institute of Biotechnology, Nantong, China), protein concentrations were detected using a bicinchoninic acid assay kit (Beyotime Institute 

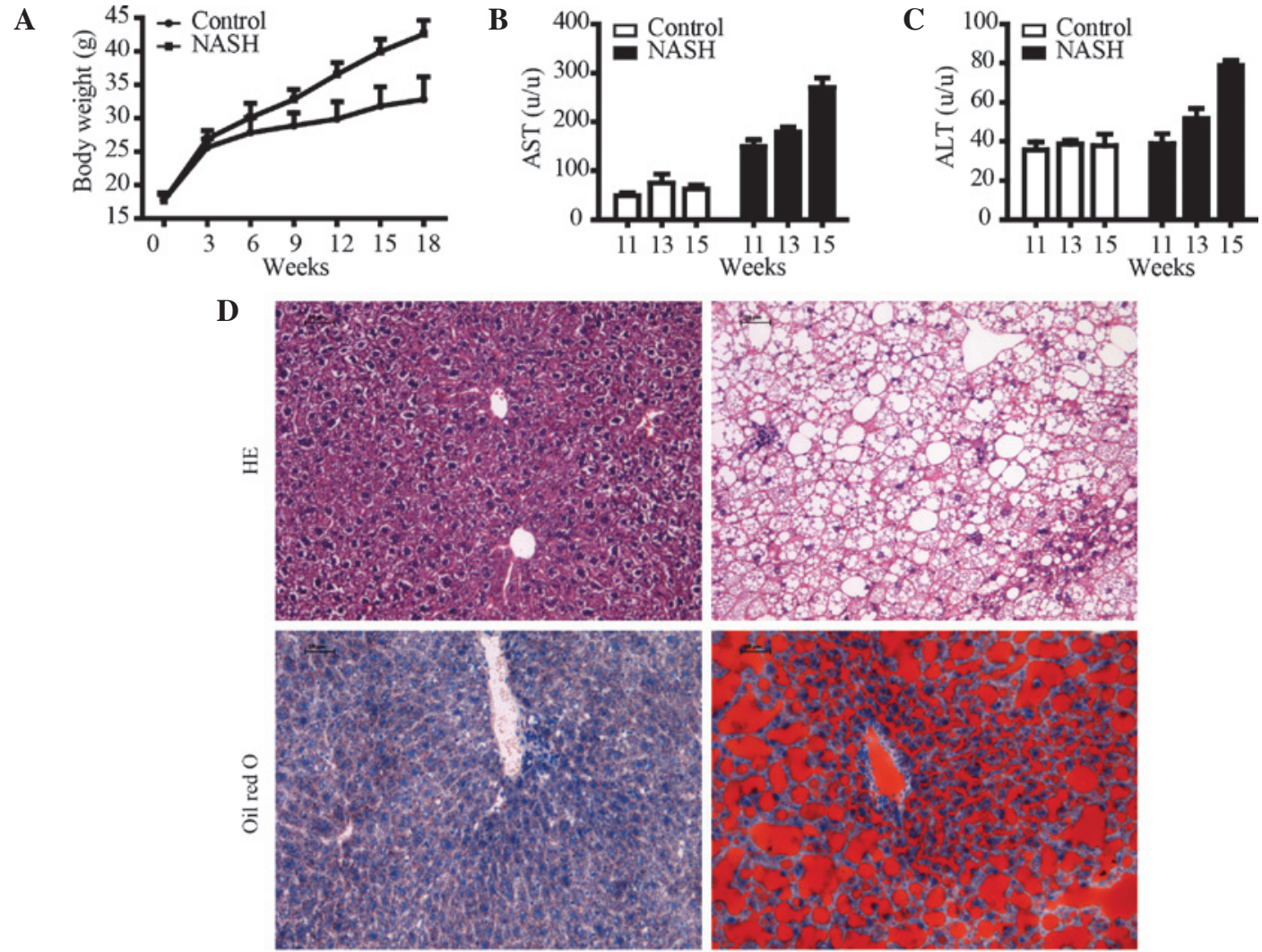

Control

NASH

Figure 1. Physiological and biochemical parameters, and histological analysis of mice that were fed a HFD to induce NASH. (A) The body weight was monitored weekly during intake of the HFD. Values are expressed as the mean \pm standard deviation $(n=20)$. (B and C) Activities of ALT and AST were detected at weeks 11, 13, 15 of HFD. Values are expressed as the mean \pm standard deviation ( $\mathrm{n} \geq 6$ in each group). (D) Hepatic morphological changes were examined microscopically following HE staining and hepatic lipid accumulation was examined by Oil Red O staining at 18 weeks of intake of HFD. Representative images of each group are shown (magnification, x40). NASH, non-alcoholic steatohepatitis; HFD, high-fat diet; HE, hematoxylin and eosin; AST, aspartate aminotransferase; ALT, alanine aminotransferase.
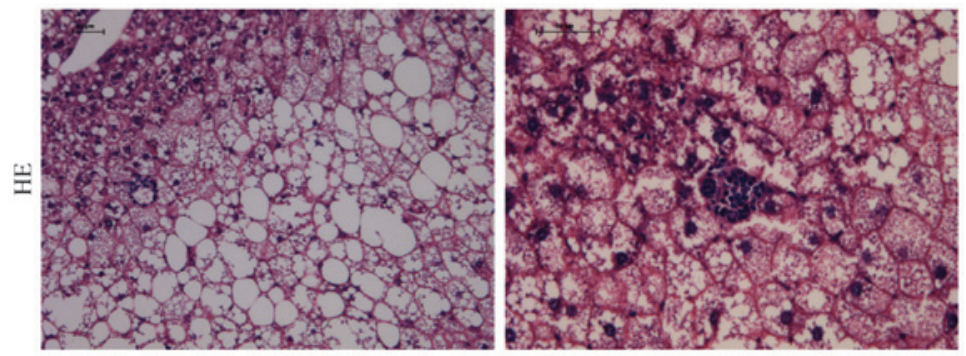

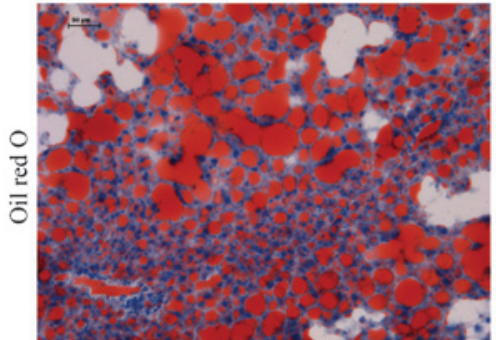

Normal saline

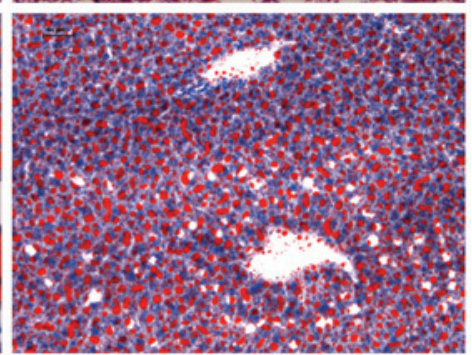

Nicotine

Figure 2. Activation of the $\alpha 7$-nicotinic acetylcholine receptor by nicotine treatment attenuated high-fat-diet-induced hepatic steatosis in mice. Representative HE-stained and Oil Red O-stained liver sections are shown (magnification, x40). HE, hematoxylin and eosin.

of Biotechnology) and western blotting was performed, as previously described (27). Briefly, equal amounts of protein were separated by SDS-PAGE. Proteins were then transferred onto nitrocellulose membranes and identified with anti- $\alpha 7 \mathrm{nAChR}$ polyclonal antibody (cat. no. 23791-AP; Proteinch USA), anti-NF- $\kappa$ B monoclonal antibody (cat. no. 4764S; Cell Signaling Technology, Inc., Beverly, MA, USA), anti-inhibitor of NF- $\kappa \mathrm{B}$ (IкB) antibody (cat. no. 4814; CST Company, Boston, MA, 
A

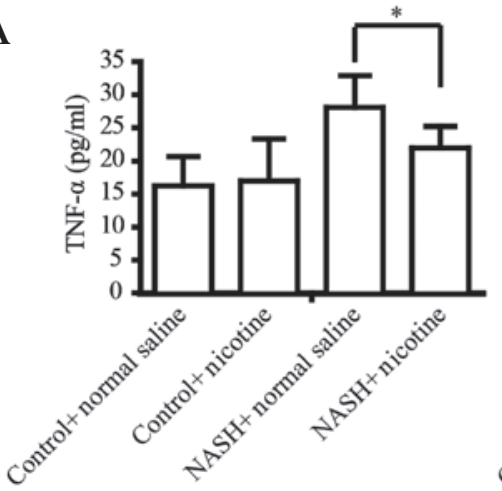

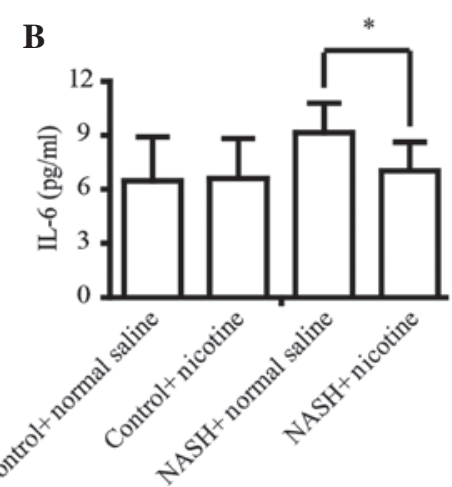

Figure 3. Nicotine induces suppression of TNF- $\alpha$ and IL-6 in mouse model of non-alcoholic fatty liver disease. Mice were treated with nicotine (5 mg/kg, intraperitoneally) or vehicle for three weeks, and the levels of (A) TNF- $\alpha$ and (B) IL-6 were measured. Values are expressed as the mean \pm standard deviation ( $\mathrm{n}=9$ per group). ${ }^{*} \mathrm{P}<0.05$. TNF, tumor necrosis factor; IL, interleukin; NASH, non-alcoholic steatohepatitis.

USA), anti-extracellular signal-regulated kinase (ERK) monoclonal antibody (cat.no. 20G11; Cell Signaling Technology, Inc.) and anti-GAPDH antibodies (Santa Cruz Biotechnology, Inc., Dallas, TX, USA) at 1:1,000. Detection was performed using a horseradish peroxidase-conjugated secondary antibody and SuperSignal West Pico Chemiluminescent Substrate (Pierce Biotechnology, Inc., Rockford, IL, USA) according to the manufacturer's instructions. Kodak films (Kodak, Rochester, NY, USA) were used to visualize the gels.

Statistical analysis. Values are expressed as the mean \pm standard deviation. Statistical analyses were performed using one-way analysis of variance or the unpaired Student's $t$-test as indicated. Statistical analysis was performed using SPSS v.10.0 statistical software (SPSS, Inc., Chicago, IL, USA). $\mathrm{P}<0.05$ was considered to indicate a statistically significant difference between values.

\section{Results}

HFD-induced NASH. In the present study, a mouse model of NASH was generated by intake of a HFD. After 18 weeks of HFD intake, the body weight was significantly increased, which indicated the establishment of the obesity mouse model (Fig. 1A). As shown in Fig. 2B and C, activities of AST and ALT were increased in mice on an HFD compared with those in the control mice which received a normal diet. It appeared that the HFD induced liver injury. To determine whether HFD induced hepatic steatosis, liver pathological examination by H\&E staining was performed (Fig. 1D). The hepatic cell structure in the control group was normal. However, the HFD increased hepatic damage with obvious hepatic necrosis. Further examination of the hepatic lipid accumulation status with Oil Red O staining revealed that the HFD significantly induced hepatic lipid accumulation compared to that in the control group.

Activation of $\alpha 7$ nAChR attenuates HFD-induced hepatic steatosis. In order to identify whether activation of $\alpha 7 \mathrm{nAChR}$ can prevent NASH and the subsequent hepatic injury, $\alpha 7 \mathrm{nAChR}$ agonist nicotine was administered to mice receiving the HFD. As shown in Fig. 2, administration of nicotine significantly, but not completely, prevented HFD-induced hepatic necrosis and hepatic lipid accumulation.
A
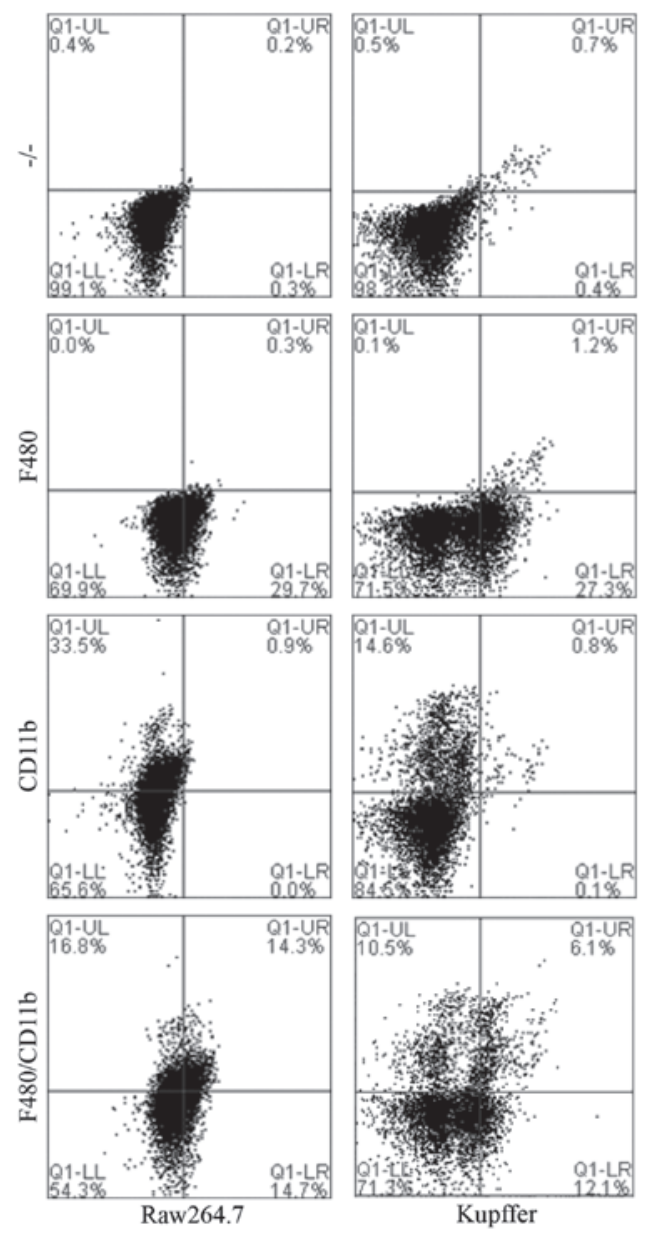

B

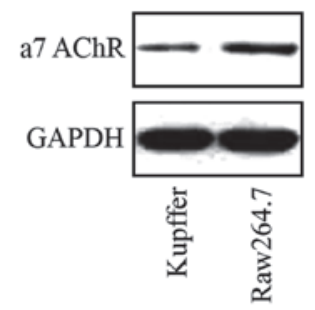

Figure 4. Isolated and identified primary liver Kupffer cells. (A) The isolated primary liver Kupffer cells were identified by using flow cytometry and compared with RAW264.7 cells. (B) $\alpha 7 \mathrm{nAChR}$ expression profiles in primary liver Kupffer cells and RAW264.7 cells. GAPDH was employed as a loading control. Results shown are representative of at least three independent experiments. $\alpha 7 \mathrm{nAChR}, \alpha 7$-nicotinic acetylcholine. 

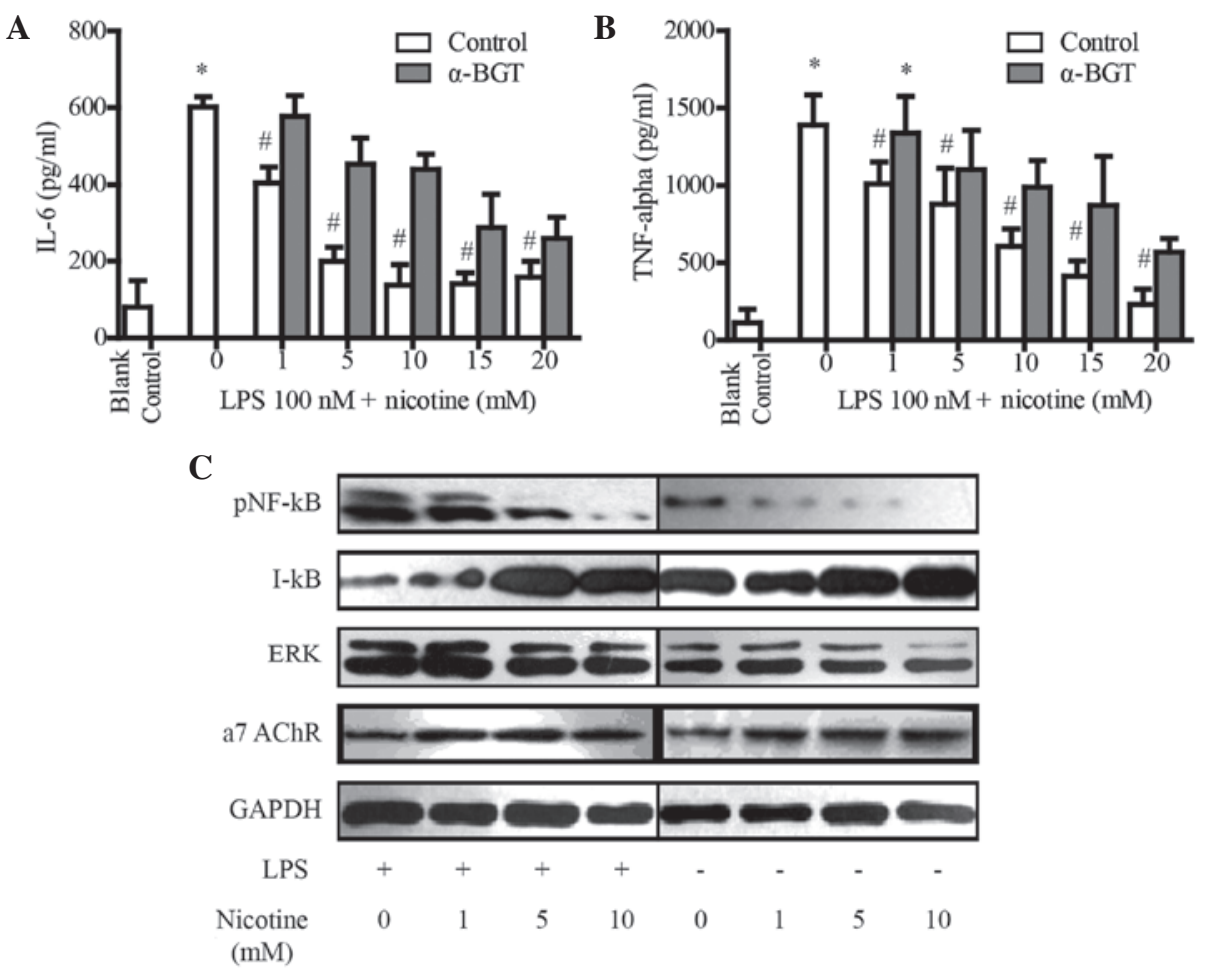

Figure 5. Nicotine reduces the secretion of TNF- $\alpha$ and IL-6 in primary Kupffer cells via $\alpha 7$ nAChR. (A and B) The supernatant was collected from the culture of Kupffer cells after treatment with LPS and nicotine in the presence or absence of $\alpha$-BGT, and cytokine levels were assessed using ELISA kits. Values are expressed as the mean \pm standard deviation of three independent experiments. ${ }^{*} \mathrm{P}<0.01$ vs. blank control group; ${ }^{*} \mathrm{P}<0.01 \mathrm{vs}$. LPS $+0 \mathrm{nM}$ nicotine group. (C) Effects of nicotine on the NF- $\mathrm{B}$ and ERK pathway in primary Kupffer cells. GAPDH was used as a loading control. Blots shown are representative of at least three independent experiments. TNF, tumor necrosis factor; IL, interleukin; NF- $\kappa \mathrm{B}$, nuclear factor kappa B; p, phosphorylated, I- $\kappa \mathrm{B}$, inhibitor of NF- $\kappa$; ERK, extracellular signal-regulated kinase; $\alpha 7 \mathrm{nAChR}, \alpha 7$-nicotinic acetylcholine; LPS, lipopolysaccharide; $\alpha$-BGT, $\alpha 7 \mathrm{nAChR}$ antagonist $\alpha$-bungarotoxin.

Activation of $\alpha 7$ nAChR attenuates HFD-induced hepatic inflammation. Inflammation is the main pathological consequence of HFD-induced NASH and is characterized by a release of inflammatory factors, which contributes to hepatic fibrosis $(28,29)$. Thus, the present study determined whether nicotine can prevent HFD-induced hepatic inflammation. The secretion of the classic inflammatory factors TNF- $\alpha$ and IL-6 was detected by ELISA. The HFD significantly upregulated the serum levels of TNF- $\alpha$ and IL-6 in mice. However, nicotine treatment significantly attenuated HFD-induced upregulation of serum TNF- $\alpha$ and IL-6 (Fig. 3).

Nicotine exerts anti-inflammatory effects via targeting a7 $n A C h R$ and inhibiting the NF- $\kappa B$ and ERK pathways. To investigate the underlying mechanism of the reduction of pro-inflammatory cytokines TNF- $\alpha$ and IL- 6 by $\alpha 7 \mathrm{nAChR}$ activation, primary macrophages from the liver were isolated and assessed. Primary liver Kupffer cells were successfully isolated and identified by staining with CD11b and F480 macrophage-specific markers (Caltag Laboratories, Burlingame, CA, USA) and flow cytometric detection (BD-Accuri C6, BD Biosciences Franklin Lakes, NJ, USA) in comparison with murine macrophage RAW 264.7 cells (Fig. 4).

In the mouse model of NASH, the $\alpha 7 \mathrm{nAChR}$ agonist nicotine significantly attenuated HFD-induced upregulation of serum TNF- $\alpha$ and IL-6. To determine the anti-inflammatory mechanisms of $\alpha 7 \mathrm{nAChR}$-activation, the present study assessed whether nicotine treatment blocked the production of TNF- $\alpha$ and IL- 6 with or without $\alpha 7 \mathrm{nAChR}$ antagonist $\alpha$-bungarotoxin
$(\alpha$-BGT) in the primary liver Kupffer cells. The secretion of the classic inflammatory factors TNF- $\alpha$ and IL-6 was detected by ELISA. Stimulation with lipopolysaccharide (LPS) significantly upregulated the secretion of TNF- $\alpha$ (Fig. 5A) and IL-6 (Fig. 5B) in the cell culture supernatants. However, nicotine treatment significantly attenuated LPS-induced upregulation of TNF- $\alpha$ (Fig. 5A) and IL-6 (Fig. 5B) in a dose-dependent manner. Furthermore, $\alpha 7 \mathrm{nAChR}$ antagonist $\alpha$-BGT blocked the nicotine-induced reduction of TNF- $\alpha$ (Fig. 5A) and IL-6 (Fig. 5B), which indicated that nicotine reduced the production of TNF- $\alpha$ and IL- 6 via activating $\alpha 7 \mathrm{nAChR}$.

Release of inflammatory cytokines is mostly mediated via the ERK and NF- $\kappa B$ pathways, and ERK and NF- $\mathrm{KB}$ are the main downstream signaling molecules of $\alpha 7 \mathrm{nAChR}$ (27). The present study therefore investigated whether activation of $\alpha 7 \mathrm{nAChR}$ reduces the production of cytokines via inhibiting the ERK and NF- $\kappa B$ pathways (Fig. 5C). As shown in Fig. 5, nicotine treatment upregulated the protein levels of $\alpha 7 \mathrm{nAChR}$ in Kupffer cells in a dose-dependent manner, which was consistent with the results of a previous study (27). Furthermore, nicotine obviously downregulated ERK and NF- $\mathrm{KB}$ levels in Kupffer cells.

\section{Discussion}

The results present study suggested that specific interference with $\alpha 7 \mathrm{nAChR}$ represents a novel strategy for the treatment of NASH. It was shown that treatment with the $\alpha 7 \mathrm{nAChR}$ agonist nicotine for three weeks obviously attenuated hepatic 
steatosis and reduced the production of TNF- $\alpha$ and IL- 6 in an HFD-induced mouse model of NASH. To investigate the underlying mechanism, the primary macrophages from mouse livers were isolated and treated with nicotine. The results showed that nicotine reduced LPS-induced secretion of TNF- $\alpha$ and IL- 6 in vitro, which was blocked by $\alpha 7 \mathrm{nAChR}$ antagonist $\alpha$-BGT. These results indicated that nicotine suppressed TNF- $\alpha$ and IL- 6 secretion by LPS-stimulated macrophages through $\alpha 7 \mathrm{nAChR}$ activation. Furthermore, the present study showed that nicotine-stimulated $\alpha 7 \mathrm{nAChR}$ activation significantly downregulated NK- $\kappa \mathrm{B}$ and ERK. It appeared that the activation of $\alpha 7 \mathrm{nAChR}$ suppressed the production of pro-inflammatory cytokines through NK- $\kappa \mathrm{B}$ and ERK pathways.

$\mathrm{NASH}$ is increasingly recognized as a major epidemiological problem, linking the metabolic syndrome to liver fibrosis, cirrhosis and hepatocellular carcinoma. Currently discussed treatment options comprise drugs approved for managing the symptoms of impaired glucose metabolism, hypertension and hyperlipidemia, including angiotensin I antagonists or insulin sensitizers (30). However, the incidence of NAFLD in the human population is further increasing, affecting up to $30 \%$ of the general population worldwide, despite the availability of these drugs (31). In addition, the side effects of approved drugs preclude treatment of patient sub-populations, thus underlining the requirement for additional specific treatment options (27).

To test the effects of $\alpha 7 \mathrm{nAChR}$ activation on $\mathrm{NASH}$, the HFD-induced mouse model of NASH was employed. The model developed symptoms within a time frame of 18 weeks and was characterized by a rather mild elevation in liver enzymes, such as ALT, in the circulation as well as the presence of lobular inflammation, which is also observed in humans with NASH (32). The present study showed that the $\alpha 7 \mathrm{nAChR}$ agonist nicotine reduced NASH-associated hepatic steatosis in mouse models. Furthermore, nicotine treatment decreased the secretion of the pro-inflammatory cytokines TNF- $\alpha$, IL-6 in mice with NASH. This result indicated that activation of $\alpha 7 \mathrm{nAChR}$ and the resulting anti-inflammatory effects may represent a novel therapeutic strategy for NASH.

Inflammation characterized by the release of soluble factors, including chemokines and cytokines, in addition to immune cell activation, is regarded as an integral part of NASH and several lines of evidence suggested that targeting of inflammation is a promising tool for the management of NASH (29). The results of the present study showed that a7 $\mathrm{nAChR}$ agonist nicotine reduced the production of TNF- $\alpha$ and IL- 6 in the mouse model of NASH in vivo and in primary Kupffer cells in vitro. These results were consistent with those of previous studies, which reported that activation of the a7 $\mathrm{nAChR}$ expressed on resident macrophages may suppress the local inflammation by reducing the production of pro-inflammatory cytokines TNF- $\alpha$ and IL-6 (27). Furthermore, the present study found that nicotine-induced a7 $\mathrm{nAChR}$ activation significantly inhibited the expression of $\mathrm{NF}-\kappa \mathrm{B}$ and ERK. This result indicated that activation of the a7 $\mathrm{nAChR}$ may inhibit cytokine production by Kupffer cells via the NF- $\mathrm{NB}$ and ERK pathways.

In conclusion, the present study indicated that modulating the inflammatory response in affected livers via activating a7 $\mathrm{nAChR}$ may represent a novel strategy for the treatment of NASH. The feasibility of this strategy requires pre-clinical and clinical validation in further studies.

\section{Acknowledgements}

This work was supported by the National Natural Science Foundation of China (grant no. 81100279) and the Foundation of Zhejiang Health Committee (no. 2013KYA145).

\section{References}

1. Noureddin M, Yates KP, Vaughn IA, Neuschwander-Tetri BA, Sanyal AJ, McCullough A, Merriman R, Hameed B, Doo E, Kleiner DE, et al: Clinical and histological determinants of nonalcoholic steatohepatitis and advanced fibrosis in elderly patients. Hepatology 58: 1644-1654, 2013.

2. Evans CD, Oien KA, MacSween RN and Mills PR: Non-alcoholic steatohepatitis: A common cause of progressive chronic liver injury?. J Clin Pathol 55: 689-692, 2002.

3. Ascha MS, Hanouneh IA, Lopez R, Tamimi TA, Feldstein AF and Zein NN: The incidence and risk factors of hepatocellular carcinoma in patients with nonalcoholic steatohepatitis. Hepatology 51: 1972-1978, 2010.

4. Adams LA, Lymp JF, St Sauver J, Sanderson SO, Lindor KD, Feldstein A and Angulo P: The natural history of nonalcoholic fatty liver disease: A population-based cohort study. Gastroenterology 129: 113-121, 2005.

5. Dunn W, Xu R, Wingard DL, Rogers C, Angulo P, Younossi ZM and Schwimmer JB: Suspected nonalcoholic fatty liver disease and mortality risk in a population-based cohort study. AM J Gastroenterol 103: 2263-2271, 2008.

6. Wong VW, Wong GL, Tsang SW, Fan T, Chu WC, Woo J, Chan AW, Choi PC, Chim AM, Lau JY, et al: High prevalence of colorectal neoplasm in patients with non-alcoholic steatohepatitis. Gut 60: 829-836, 2011.

7. Gastaldelli A, Kozakova M, Højlund K, Flyvbjerg A, Favuzzi A, Mitrakou A and Balkau B: Fatty liver is associated with insulin resistance, risk of coronary heart disease and early atherosclerosis in a large European population. Hepatology 49: 1537-1544, 2009.

8. Bilici A, Ozguroglu M, Mihmanli I, Turna H and Adaletli I: A case-control study of non-alcoholic fatty liver disease in breast cancer. Med Oncol 24: 367-371, 2007.

9. Pagadala MR, Zein CO, Dasarathy S, Yerian LM, Lopez R and McCullough AJ: Prevalence of hypothyroidism in nonalcoholic fatty liver disease. Dig Dis Sci 57: 528-534, 2012.

10. Caballería L, Auladell MA, Torán P, Miranda D, Aznar J, Pera G, Gil D, Muñoz L, Planas J, Canut S, et al: Prevalence and factors associated with the presence of non alcoholic fatty liver disease in an apparently healthy adult population in primary care units. BMC Gastroenterol 7: 41, 2007.

11. Gelpi Méndez JA, Castellanos Fillot A, Sainz Gutiérrez JC, Quevedo Aguado L and Martin Barallat J: Prevalence of non-alcoholic fatty liver disease and associated risk factors among managers from the community of Madrid. Arch Prev Riesgos Labor 17: 84-90, 2014 (In Spanish).

12. Leite NC, Salles GF, Araujo AL, Villela-Nogueira CA and Cardoso CR: Prevalence and associated factors of non-alcoholic fatty liver disease in patients with type-2 diabetes mellitus. Liver Int 29: 113-119, 2009.

13. Loria P, Lonardo A, Lombardini S, Carulli L, Verrone A, Ganazzi D, Rudilosso A, D'Amico R, Bertolotti M and Carulli N: Gallstone disease in non-alcoholic fatty liver: Prevalence and associated factors. J Gastroen Hepatol 20: 1176-1184, 2005.

14. Radu C, Grigorescu M, Crisan D, Lupsor M, Constantin D and Dina L: Prevalence and associated risk factors of non-alcoholic fatty liver disease in hospitalized patients. J Gastrointestin Liver Dis 17: 255-260, 2008.

15. Hu KC, Wang HY, Liu SC, Liu CC, Hung CL, Bair MJ, Liu CJ, Wu MS and Shih SC: Nonalcoholic fatty liver disease: Updates in noninvasive diagnosis and correlation with cardiovascular disease. World J Gastroenterol 20: 7718-7729, 2014.

16. Sanyal AJ: NASH: A global health problem. Hepatol Res 41: 670-674, 2011.

17. Takaki A, Kawai D and Yamamoto K: Molecular mechanisms and new treatment strategies for non-alcoholic steatohepatitis (NASH). Int J Mol Sci 15: 7352-7379, 2014.

18. Musso G, Anty R and Petta S: Antioxidant therapy and drugs interfering with lipid metabolism: could they be effective in NAFLD patients? Curr Pharm Des 19: 5297-5313, 2013. 
19. Yalcin M, Akarsu M, Celik A, Sagol O, Tunali S, Ertener O, Bengi $G$ and Akpinar $H$ : A comparison of the effects of infliximab, adalimumab, and pentoxifylline on rats with nonalcoholic steatohepatitis. Turk J Gastroenterol. 25: 167-175, 2014.

20. Fock KM and Khoo J: Diet and exercise in management of obesity and overweight. J Gastroenterol Hepatol 28: 59-63, 2013.

21. Beaton MD: Current treatment options for nonalcoholic fatty liver disease and nonalcoholic steatohepatitis. Can J Gastroenterol 26 : 353-357, 2012.

22. Malaguarnera M, Di Rosa M, Nicoletti F and Malaguarnera L: Molecular mechanisms involved in NAFLD progression. J Mol Med (Berl) 87: 679-695, 2009.

23. Satapathy SK, Garg S, Chauhan R, Sakhuja P, Malhotra V, Sharma BC and Sarin SK: Beneficial effects of tumor necrosis factor-alpha inhibition by pentoxifylline on clinical, biochemical and metabolic parameters of patients with nonalcoholic steatohepatitis. AM J Gastroenterol 99: 1946-1952, 2004.

24. Mitchel EB and Lavine JE: Review article: the management of paediatric nonalcoholic fatty liver disease. Aliment Pharmacol Ther 40: 1155-1170, 2014.

25. Filippini P, Cesario A, Fini M, Locatelli F and Rutella S: The Yin and Yang of non-neuronal $\alpha 7$-nicotinic receptors in inflammation and autoimmunity. Curr drug targets 13: 644-655, 2012.
26. Boeckxstaens G: The clinical importance of the anti-inflammatory vagovagal reflex. Handb Clin Neurol 117: 119-134, 2013.

27. Ganz M and Szabo G: Immune and inflammatory pathways in NASH. Hepatol int 7: 771-781, 2013.

28. Meli R, Mattace Raso G and Calignano A: Role of innate immune response in non-alcoholic Fatty liver disease: metabolic complications and therapeutic tools. Front Immunol 5: 177, 2014.

29. Braunersreuther V, Viviani GL, Mach F and Montecucco F: Role of cytokines and chemokines in non-alcoholic fatty liver disease. World J Gastroenterol 18: 727-735, 2012.

30. Weiß J, Rau M and Geier A: Non-alcoholic fatty liver disease epidemiology, clinical course, investigation, and treatment. Dtsch Arztebl Int 111: 447-452, 2014.

31. Ratziu V, Goodman Z and Sanyal A: Current efforts and trends in the treatment of NASH. J Hepatol 62: S65-S75, 2015.

32. Gadd VL, Skoien R, Powell EE, Fagan KJ, Winterford C, Horsfall L, Irvine $\mathrm{K}$ and Clouston AD: The portal inflammatory infiltrate and ductular reaction in human nonalcoholic fatty liver. Hepatology 59: 1393-1405, 2014. 